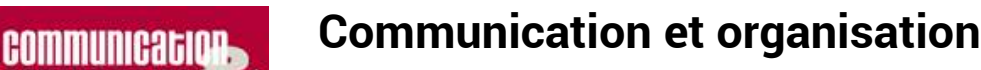

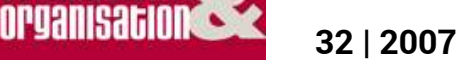

La ville dans tous les sens

\title{
Technopolis ou les paradoxes de la visibilité
}

\section{Talya Bigio}

\section{OpenEdition}

Journals

Édition électronique

URL : http://journals.openedition.org/communicationorganisation/276

DOI : 10.4000/communicationorganisation.276

ISSN : 1775-3546

\section{Éditeur}

Presses universitaires de Bordeaux

\section{Édition imprimée}

Date de publication : 1 décembre 2007

Pagination : 40-51

ISSN : 1168-5549

\section{Référence électronique}

Talya Bigio, «Technopolis ou les paradoxes de la visibilité », Communication et organisation [En ligne],

32 | 2007, mis en ligne le 01 décembre 2010, consulté le 20 avril 2019. URL : http://

journals.openedition.org/communicationorganisation/276; DOI : 10.4000/

communicationorganisation.276 
Dossier : La ville dans tous les sens

\title{
Résumé
}

L'urbanisation rapide du monde est un enjeu planétaire. Cet enjeu s'inscrit dans la mondialisation, c'est-à-dire dans le processus historique par lequel les individus accroissent leurs échanges matériels ou immatériels, à l'échelle de la planète. Les nouvelles technologies, supports de ces échanges invisibles, modifient notre relation au temps urbain. Le proche et le lointain, le dehors et le dedans, l'espace privé et l'espace public, s'interpénètrent. Ce qui pose la question de l'évolution des formes spatiales et sociales de la cité. Liés aux usages des objets communicants, de nouveaux comportements individuels au milieu d'inconnus apparaissent. Mais aussi, de nouvelles formes ritualisées de rencontre. L'intime devient public. D'un côté, on assiste au développement d'une société de surveillance, panoptique. De l'autre, au développement du festif généralisé. A partir de l'art urbain, il s'agit d'analyser le positionnement de l'individu face à un environnement devenu médiatique.

\section{Mots-clés}

TIC, media, urbanité, éthique, espace public, art urbain, publicité

\begin{abstract}
The fast urbanization of today's world is a planetary challenge. This challenge is part of globalisation i.e in the historical process in which people increase the material or immaterial exchange at the scale of the planet. New technologies, which is the base of these immaterial exchanges, modify our comprehension of time. Time and space, the near and the far, the outside and the inside, all mix together. Linked to the use of objects of communication, new behaviours appear one is admits unknown people. But also, new ritualiazed types of meeting. Privacy becomes public. On one hand a «Big Brother » type of society, which permits a visibility of each of us by all. On the other hand, we see a development of generalized festivity activities. We must analyzed, based on urban art, the behaviour of the individual, in an environment which has become mediatise.
\end{abstract}

\section{Key-Words}

TIC, media, urbanity, ethic, public space, urban art, publicity

Talya Bigio est architecte et diplômée en philosophie. Consultante pour la maîtrise d'ouvrage de l'état ou pour la maîtrise d'œuvre, elle anime les groupes de recherche pluridisciplinaires autour des projets architecturaux et urbains. Co-fondatrice de la rubrique architecture des Lettres Françaises, elle a écrit de nombreux articles sur l'art et la ville et a participé à plusieurs rencontres, en particulier « Métropole et Modernité », un colloque sur Walter Benjamin organisé par le Collège international de philosophie. 


\section{Technopolis ou les paradoxes de la visibilité Talya Bigio}

talyabi@yahoo.fr

L'apparition des nouvelles technologies de communication et d'information entraîne une crise des médias. Celle-ci est à la fois une crise des pouvoirs, et une crise de la culture. C'est une crise des pouvoirs, car elle transforme la nature du débat démocratique dans la cité, les possibilités d'action dans l'espace public. C'est une crise de la culture, car les nouveaux réseaux entraînent une dé-réalisation de la dimension publique de l'espace urbain et développent de nouveaux modèles de spectacularisation du monde.

Les nouvelles technologies, qui accroissent la possibilité de voir l'autre à distance instaurent, paradoxalement, une vision intime dans l'espace public. Elles transforment la notion d'unité de voisinage. Une nouvelle urbanité se met en place qui modifie l'enjeu spatial concret de la place, de l'édifice ou de la rue. Cet enjeu, qui est l'objet de notre étude, est d'ordre éthique : c'est la question de la rencontre avec l'autre dans un espace public désormais médiatisé. Nous tenterons de l'examiner à travers différentes thématiques :

- l'évolution de la ville contemporaine vers davantage de surveillance et le développement d'une société de divertissement ;

- l'imaginaire de la ville à partir de la publicité et de l'art urbain ;

- les transformations de la communication entre les individus liées aux TIC ou aux objets communicants : la projection de l'intime, la création d'un dedans du dehors, la confusion des autres et de soi.

La " ville-paradoxe ": théâtre d'une société du divertissement ou d'une société de surveillance

Dans la technopolis, la question de la communication est dialectique. D'un côté, elle est ouverte par les immenses possibilités d'expression du web. De l'autre, la planétarisation de la technique opère une fonction de contrôle et de normalisation des comportements sociaux. Aussi la ville contemporaine est-elle construite sur un paradoxe : c'est le lieu de la liberté, dans lequel l'homme peut agir ; mais c'est aussi l'espace de la surveillance et du quadrillage. La communication est un besoin et un droit. Le «droit à la ville», c'est la possibilité pour chaque individu de participer à la construction éthique ou spatiale de la cité. L'enjeu de ce droit, dans un environnement devenu médiatique, 
c'est défendre la liberté en s'appuyant sur le pouvoir-faire de la technique, plutôt qu'en s'y soumettant.

Mais l'intervention de la technique peut aussi se traduire par un quadrillage qui transforme l'espace urbain en un lieu de surveillance. Dans la ville de Londres, nul ne peut échapper au regard des caméras, qui lui demeure caché. Dans un territoire urbain transformé en un gigantesque panopticon, la maîtrise de l'environnement médiatique conditionne les libertés publiques. Il « s'agit de se sentir chez soi, libre et en sécurité », comme le suggère la publicité pour Aérospatiale qui ajoute que "savoir se défendre, c'est pouvoir être libre». Le risque, c'est qu'au nom de la légitime défense ou de la conservation, la société ne se transforme en une société de surveillance, dans laquelle chacun surveille chacun. Ce risque est accru par l'avancée des nouvelles technologies, qui amplifient les possibilités d'identification d'un individu à partir de ses traces ADN.

Désormais, le contrôle de nos traces nous échappe, que ce soit les traces biologiques ou celles de notre vie privée. Le logiciel «speak on » diffuse sans autorisation les portraits de vie privée. Le monde de la technosurveillance s'enracine aussi dans le système invisible des échanges sur le Web. L'envers de la société de surveillance, qui décloisonne les frontières entre vie privée et vie publique, c'est la société du tourisme ou du festif généralisé. La ville y est vécue sur le mode d'un terrain de jeu que le citadin peut parcourir en tous sens. Le réseau internet devient alors le support d'actions collectives éphémères. Une mise en œuvre dans l'espace physique de la ville, des rencontres virtuelles.

La ville est interprétée, comme elle l'avait été par les situationnistes, comme un terrain de jeu participatif et festif. Avec les « expériences psycho-géographiques », il s'agissait d'unir l'art à la vie, et de libérer celle-ci par la poésie, la psychanalyse ou le jeu avec l'architecture. Les actuelles errances urbaines de l'art construisent une poétique de la ville conçue comme une succession de rencontres et d'actes. Une théâtralité de l'intime peut être déclenchée de manière intempestive sur internet, comme la scène du baiser qui a rassemblé des centaines d'internautes sous la Grande Arche de la Défense, ou plus récemment, le dîner en blanc place de l'Etoile, au pied de l'Arc de Triomphe. Des scénographies urbaines de l'art prolongent cette poétique de l'espace, comme les signatures de prénoms d'enfants handicapés sur les troncs d'arbres de la perspective du jardin du Luxembourg, recouverts par une bande de tissu de couleur. Mises en scène à partir d'une communauté de rencontre virtuelle, ou à partir d'une commande 
publique, ces actions poétiques ou artistiques s'inscrivent dans la ville historique en créant, à partir de scénarios de l'intime projetés dans l'espace de la cité, des territoires psycho-affectifs éphémères.

Dans la ville moderne, la signification de l'art naît comme évasion. Dans la ville de la technique post-moderne, à cette signification s'ajoute l'idée que le centre ou le moteur de l'action artistique, c'est l'expression de l'individu anonyme. L'idée que l'auteur d'une œuvre d'art peut être, comme l'auteur sur internet, de plus en plus difficile à identifier. La scène urbaine devient alors le théâtre de l'organisation d'une société du divertissement, structurée sur le modèle d'une communauté de partage virtuel sur internet.

\section{L'imaginaire urbain dans la publicité}

Pour Marshall Mac Luhan, la publicité est une des grandes formes de l'art au $\mathrm{XX}^{\mathrm{e}}$ siècle. Si l'art, est selon Nietzsche, une vérification du réel, l'art publicitaire met en scène un simulacre du réel. Quel est alors le rôle de la publicité dans l'élaboration d'un discours sur la ville ? La publicité tend à effacer les frontières entre réel et imaginaire, entre paysage urbain réel et fiction du paysage. C'est ainsi que la publicité contribue à faire disparaître l'image de la ville traditionnelle. Elle reconstruit le temps urbain et fait de la ville un paysage "naturel ", qui n'est plus soumis au temps historique.

La ville peut alors être regardée comme une seconde nature. La nature, comme le fond commun qui nous rassemble et qu'il s'agit de préserver. La mise en scène de l'artiste Spencer Tunick pour la campagne de Green Peace, une foule nue, vue de dos, de 600 personnes sur le glacier d'Aletsch, alerte le public. La communauté humaine fait fond sur une nature fragile qu'il s'agit de sauver. Le paradoxe soulevé par l'association entre le nu et le paysage dans l'image, c'est qu'il n'y a de construction que culturelle. Nature et culture sont liées par le regard prothétique de l'appareil photographique. A l'opposé, l'affiche de Spencer Tunick agite la fiction de l'origine et celle de l'état de nature. La publicité tend à naturaliser l'histoire. A la réflexion démocratique du lien entre les hommes à partir de leur nature politique, elle substitue la relation entre l'individu et son environnement. Dans la publicité pour la livebox, la terre est représentée comme un seul territoire, sans frontières géographiques fixes, sans distinction entre espace urbain et espace naturel. Depuis le vaisseau spatial, elle montre l'image d'une planète habitée par une communauté solidaire et joyeuse, ce qui nous permet «d'aller ensemble plus loin». Derrière l'image de naturalité de la 
planète, la publicité pour la livebox reconstruit le mythe d'une communauté universellement pacifiée.

La transformation des valeurs sociales vers le divertissement est rendu manifeste par l'évolution de la publicité pour Nike. Dans les années 90, la publicité montrait l'image d'un cycliste qui montait péniblement une montagne. La publicité privilégiait alors la volonté et le dépassement héroïque de soi. Aujourd'hui, la publicité privilégie les situations de loisir dans l'espace urbain. La publicité pour les chaussures Nike met en scène un jeune homme engagé dans une course-poursuite dans le métro. L'espace urbain est transformé en lieu de jouissance et d'action. A l'image du mode ludique des jeux vidéo, le personnage est une figure de l'éternel adolescent qui s'invente le modèle d'une cité qu'il peut librement parcourir en tous sens. La publicité pour la voiture Jeep, nous rappelle que « toutes les aventures sont au coin de la rue ». Ici, le paysage naturel ou urbain, déréalisé et numérisé, se transforme en paysage idéalisé. Dans la publicité pour Air France, l'espace commun, celui qui relie les espaces urbains entre eux, c'est l'atmosphère des hauteurs. Vécue comme dimension temporelle de l'espace et du temps, la ville est déracinée. Elle est le théâtre d'une situation de liberté comme délivrance. Il s'agit d'échapper aux contraintes de la circulation automobile. La mobilité urbaine est le vecteur de la publicité. Le slogan, «le ciel est sur la terre » évoque l'icône de Malevich, une icône concrète et non objective ayant vaincu les relations terrestres. L'avion a vaincu les frontières et a affranchi l'homme des contraintes de la pesanteur. L'œil de la caméra traverse les frontières, de la chambre à coucher à la scène urbaine. La révolution technique réalise notre rêve de bonheur, de confort et de luxe et nous installe, au sein même de l'urbain, dans un bien-être nuageux.

La publicité restitue une image déformée du réel. Soit elle naturalise notre rapport à l'histoire et fait apparaître la communauté des hommes comme une communauté naturelle qui préexiste à toute société instituée. Soit elle contribue, par le biais des techniques de synthèse, à déterritorialiser la ville. En construisant l'ordre de l'image d'une nature idéalisée et numérisée, elle occulte les relations de pouvoir socioéconomiques et fait apparaître le marché comme un ordre naturel. Elle fait rêver et incite à la consommation.

\section{L'imaginaire urbain dans l'art}

Le média publicitaire transforme l'espace commun en lieu d'appropriation subjective de l'objet culturel ou marchand. La publicité s'impose à nous, dans l'espace public, de manière inévitable. Dans un 
environnement urbain médiatisé par l'image publicitaire, la fonction de l'art se déplace. Le parcours des œuvres qui jalonnent le trajet du tramway, boulevard des Maréchaux-sud, sur une des limites urbaines de Paris, en témoigne. La proposition, installer des œuvres d'art «durables», pose la question de la tendance actuelle de l'art contemporain vers la scénographie éphémère et l'auto-fiction, et affirme le statut de l'artiste comme auteur dans l'espace public.

La ville se métamorphose en un terrain de jeux pour l'art. La commande publique propose à chaque artiste de choisir un lieu le long du parcours du tramway et de l'investir par une œuvre. Ce parcours d'œuvres se transforme alors, pour le piéton, en un jeu de piste urbain, à la rencontre des œuvres, à l'image de la navigation libre sur internet. Les artistes élaborent des scénarios du futur, qui interrogent la place de l'homme dans une ville devenue espace fonctionnel, désymbolisé par la complexité machinique.

Les œuvres disséminées le long du parcours du tramway sont des architecture-sculptures qui dialoguent avec leur environnement immédiat. La tour-totem de Didier Fiuza Faustino I.S.Q.H. est une tour solitaire en face des grands ensembles de la porte d'Ivry. Lorsqu'il n'y a plus rien à conquérir au niveau du sol, l'aménagement urbain se tourne vers de nouvelles stratégies constructives : villes du dessus qui se superposent aux villes du dessous, et dans lesquelles, littéralement, l'homme habite en l'air, suspendu entre ciel et terre. Totalement fermée aux regards extérieurs, la tour-totem pose la question de la communication et de l'individuation dans les grandes villes surpeuplées et du danger qu'il y aurait à ignorer la dimension du territoire de l'intime.

L'œuvre de Christian Boltanski est structurée autour d'une fiction de l'intime dans l'espace public. Dans le parc Montsouris, en face de la cité internationale, le dispositif de Christian Boltanski est une simple boîte noire contenant une bande-son pré-enregistrée de discours amoureux murmurés en neuf langues. Installée derrière un banc public, elle est déclenchée par les pas des promeneurs. L'installation met ici en œuvre une architecture textuelle, qui tisse des liens avec la ville à interpréter dans une polysémie des voix. L'œuvre de Christian Boltanski est fondée sur un paradoxe de la visibilité. Invisible dans l'espace public, elle questionne la place de l'œuvre d'art dans la ville et la fonction du musée. C'est un autre paradoxe de la visibilité que met en scène la machine scénique de Dan Graham. Le pavillon «From Boullée to eternity » est totalement transparent. C'est une sorte d'antipanopticon, qui ouvre à la question du temps et de l'altérité. L'intérieur 
est un habitacle réfléchissant, un «salon d'attente ». Le corps du spectateur se transforme en un territoire d'images instantanées. Le dispositif interroge les contraires : le mince, le gros, le dedans, le dehors ; l'espace privé, l'espace public, superposant images urbaines et images corporelles. L'homme, "placé en situation de loisir» est un simple témoin ou miroir des autres.

Dans la machine visuelle de Dan Graham, il n'y a ni point fixe, ni centre. Tout est mouvement pour un spectateur en mouvement qui le perçoit. L'échelle de l'œuvre, c'est ici l'échelle mobile de la subjectivation. La machine scénique de Dan Graham installe le spectateur dans un processus de vision affecté par l'art. Le face à face entre celui qui regarde et celui qui est regardé se fait à travers l'écran infra-mince d'un miroir sans tain. "Se voir, c'est se voir à travers le regard des autres ». Le regard livre ici une expérience directe de la communication. Dans la boîte optique transparente, la rencontre avec l'autre est une simple forme médiatisée de l'image. L'homme est un simple reflet des autres. Dan Graham s'inspire de la théorie sartrienne du regard et de l'échec de communication qui en résulte. Comme dans le piège du miroir de la «nausée », se regarder dans le miroir sans tain du pavillon de Dan Graham, ce n'est jamais voir que de l'autre. Le « moi » intime n'existe pas. Seul subsiste le « moi » social, le « moi » médiatique. La boîte optique transparente de Dan Graham est un jeu d'immersion dans le monde du spectacle urbain, qui modifie notre vision de la place publique, et notre regard sur les autres passants, les inconnus.

La fleur urbaine monumentale de Frank Gehry, au milieu du pont du Carigliano a, comme le musée Guggenheim à Bilbao, une fonction de «space generator» tissant des liens entre des lieux de nature très différentes. C'est un objet architectural «autonome», qui met en mouvement l'environnement urbain, à l'opposé d'une architecture contextuelle, qui est structurée à partir de son inscription dans le territoire urbain. Avec Frank Gehry, l'architecture naît d'un rêve, comme une architecture " impossible», dont l'inscription dans le réel urbain est le jeu et la trace. L'architecture est à l'image d'une feuille de papier froissée, qui se transforme en plis de la subjectivité, celle de l'artiste. La construction architecturale, structurée par un logiciel informatique puissant, apparait aussi comme une forme particulière des subjectivations dans l'espace public.

Nouvelle porte d'entrée de la capitale, la fleur urbaine de Frank Gehry est une œuvre de $5 \mathrm{~m}$ de hauteur, en face de la statue de la liberté. Déchirée et éclatée, c'est un manifeste des formes libres et ouvertes en 
architecture. Située au croisement du fleuve et des voies de circulation rapide, c'est à la fois un point d'ancrage et une figure du déplacement.

C'est une juxtaposition d'éléments distincts, de couleur différente, dans l'esprit des collages de Raoul Rauschenberg. Avec la fleur urbaine, la nature est soumise au regard prothétique de l'art. Résolument ancrée dans le bitume, elle réalise le mariage de la nature et de l'industrie, de la nature et de la technique, s'opposant à la nostalgie de l'exposition in situ à Paris Tar Roses de Guy Oppenheim, un des fondateurs du land art, dont les roses, aux formes pleines et entières, étaient dévorées par le bitume.

L'architecture de la fleur est d'un côté, une figure du mouvement, de l'accélération et de la vitesse. De l'autre côté, on peut pénétrer à l'intérieur, s'arrêter, voir au travers. Fondée sur une visibilité paradoxale, la sculpture-architecture institue une nouvelle symbolique urbaine. Si elle est un repère visible de loin, par tous, dans l'espace de la cité, c'est aussi une architecture invisible, fondée sur l'organisation et l'interaction avec les nouveaux réseaux de communication. Comme le musée de Bilbao, la fleur de Frank Gehry est un objet qui s'inscrit à la frontière géographique de la ville. Euvre-frontière, la fleur est aussi une installation in situ autour du téléphone urbain de Sophie Calle.

Avec cette œuvre, la question qui se pose est double : celle des limites de la ville, et celle de l'architecture, modifiée, perturbée, augmentée par l'inscription des réseaux de communication.

\section{Multipolarité des réseaux et fabrique de la ville}

Avec les nouvelles technologies, la ville contemporaine évolue vers l'incarnation d'une technologie «sans frontières » qui suscite des images contradictoires. Afin de répondre aux nécessités urbaines galopantes, des projets hors norme émergent. Certains s'inspirent des projets d'architecture futuriste. Le projet de théâtre total de MoholyNagy sert de modèle aux utopies urbaines de Beijing Boom Tower ou de DJ Rail, un train à grande vitesse qui doit enserrer la ville de Pékin, avec pour mode d'accès un tapis roulant à lévitation ultra-rapide. L'architecture est ici fabriquée autour d'une perspective numérique sans matière, sans profondeur et sans durée, pour un homme générique et abstrait dont le corps est d'abord soumis aux lois de la vitesse. Cette théâtralisation de l'espace public à l'échelle de projets monumentaux transforme la figure de l'imaginaire urbain et celle des enjeux de la communication. 
La question de la ville aujourd'hui englobe nécessairement une réflexion sur les enjeux de la structuration matérielle ou sensible de l'espace urbain à partir des TIC.

Les villes, traversées par les voies à circulation rapide ou par les autoroutes de l'information ne sont plus structurées seulement par une rencontre physique des corps dans les places et dans les rues. Le critère des flux, en se superposant aux critères géographiques transforme le temps urbain, désormais centré sur l'immédiateté. L'architecture, transformée en objet communicant autonome, s'organise autour de la nouvelle forme de centralité qu'est le temps virtuel. La fonction du mur urbain se transforme. Il devient un gigantesque écran d'affichage, aux contenus informationnels multiples, aujourd'hui majoritairement publicitaires, demain qui ouvrira un dialogue personnel avec chaque individu. Les recherches sur les écrans O.L.E.D., (électrodes à diodes électroluminescentes) transforment n'importe quel miroir en espace d'affichage, voire en espace d'interactivité grâce aux nouvelles techniques de communication, qui permettent d'exercer un contrôle à distance.

Dans un espace commun devenu médiatique, l'espace de l'intime n'est plus protégé. La limite entre espace privé et espace public tend à disparaître, créant une interpénétration entre espace de l'individu et espace des autres. L'intime devient public, spectaculaire.

\section{Objets communicants et projection de l'intime}

Une nouvelle attitude visuelle, associée aux nouvelles technologies est en train d'émerger qui transforme l'inscription de l'espace intime dans l'espace social. Les nouvelles techniques de communication dessinent une nouvelle manière d'entrer en relation avec les autres dans l'espace urbain, fondée sur la connectivité et la mobilité.

De nouveaux comportements au milieu d'inconnus apparaissent liés aux usages des objets communicants. Certaines font l'économie de l'altérité ou de l'intersubjectivité, c'est-à-dire de la rencontre avec les autres dans la réalité urbaine, réduisant celle-ci à une simple coprésence. Le téléphone mobile personnel va désormais servir de poste de télévision miniature, utilisable dans les transports en commun. Conçu pour le métro de Tokyo, le «téléglass », une prothèse visuelle fixée sur un seul œil, doit permettre selon ses concepteurs, de «transformer l'enfer du métro en expérience paradisiaque ». Ce nouvel usage, nommé «watch», installe une nouvelle attitude communautaire. Les objets communicants fabriquent une nouvelle géographie du corps et des affects. Par leur rationalité technique, ils 
nous mettent à distance de la réalité du monde extérieur. Le risque encouru, c'est d'être enfermé à l'intérieur d'un spectacle individuel et virtuel, dans une relation de soi à soi qui ne soit pas de nature réflexive.

Les objets communicants annoncent l'ère de l'humain dans la machine. Dans une œuvre récente, l'artiste Sophie Calle met en scène la réalité de la mort elle-même. A la biennale de Venise, elle expose un film sur les «sept dernières minutes» de la vie de sa mère. La mort est transformée en spectacle émotionnel et intemporel. Filmer la mort en direct, l'exposer comme une œuvre d'art dans l'espace du musée transforme celui-ci en espace de recueillement. Cette œuvre fait écho aux tags peints sur les murs de New York sur lesquels les artistes réalisent le portrait de personnes disparues. Mais aussi à la mort filmée en direct pendant les attentats du 11 septembre 2001, et qui fut diffusée sur les chaînes de télévision du monde entier. La destruction des tours jumelles apparaît comme un simulacre; le réel, comme un imaginaire surmonté.

Dans la mort filmée en direct, le défilement de l'image est interminable, et répétable à l'infini. L'enregistrement de la vie dans la boîte noire de la caméra apparaît plus vrai que la vie elle-même. Cette approche va dans la direction des dernières recherches sur le vivant. Il s'agit, grâce aux récentes découvertes en nanotechnologies, de sortir de l'humain, d'externaliser la mémoire de l'homme et de la transférer vers une boîte noire, micro-transportable. La question de la représentation de l'autre se transforme. La projection de l'intime, de soi hors de soi, dans l'espace public, à partir des nouvelles technologies entraîne une confusion des limites entre dedans et dehors, entre soi et les autres, entre espace de «soi» et espace des «autres ». Avec la projection du dedans au dehors, il n'y a plus désormais que du dehors.

\section{Conclusion}

Penser la communication, à l'ère des nouvelles technologies, c'est poser la question de la limite entre l'un et l'autre, entre voir et être vu. La mobilité post-moderne tend à faire de la rencontre avec l'autre une pseudo-rencontre, un jeu du leurre et de l'ambiguité qui maintient l'illusion de la réalité. L'usage des objets communicants est ambivalent, dans un monde qui efface les frontières entre les sphères privées et publiques. Les nouveaux systèmes de communication génèrent une réalité virtuelle. Avec les ordinateurs, chacun peut construire l'illusion mégalomaniaque d'être en contact permanent avec le monde grâce à des boutons électroniques. Voir l'autre, à distance, 
implique une transformation radicale de la perspective, mais aussi de notre perception du monde. L'univers technicien modifie notre rapport à l'échelle de la ville, qui s'étend à la planète. Le monde de la technique construit l'illusion que l'univers est transparent. Avec Google Earth et Google Sky, il s'agit d'explorer l'espace du point de vue de la terre. Avec Google Space, le point de vue est inversé. Il s'agit de produire des images de la terre depuis la lune. L'image de la terre peut être saisie d'un seul regard, omnivoyant. Comme dans l'axonométrie, c'est un regard à distance, à travers lequel l'humain peut être regardé comme autre.

La terre, vue comme un objet, peut entrer dans un processus de gestion et de surveillance. Le projet de Google est « un projet qui peut changer le futur de l'humanité ». Les nouvelles technologies nous entraînent vers un avenir imprévisible, qui peut se transformer en figure du pouvoir. Le regard sur le futur est ici anhistorique. Il ouvre la voie à la création d'espaces publics virtuels multiples. La vision technique du monde institue un droit à la transparence, auquel il est difficile d'échapper. La question de la position de l'individu face à cet environnement médiatique est une question de la responsabilité et de la liberté. Face à un monde de la technique, gouverné par internet ou par le marché, il s'agit de construire un usage des médias qui préserve l'expression libre de la singularité dans la pluralité. Face à la destruction du rapport à la ville comme monde et comme histoire, il s'agit de mettre en œuvre les conditions d'existence d'une pensée réflexive de «l'habiter». La chute du mur de Berlin a ouvert la voie à une architecture comme communication et comme contextualisation. Mais aussi à une figure de l'urbain dans laquelle la crise de la finalité est dépassée soit sur le mode ludique, soit sur le mode d'une surveillance généralisée. Nous vivons dans un univers de simulacres du réel, mais avec une possibilité de donner un sens au monde. D'où l'importance d'une architecture qui sait inventer l'utopie dans le désert ou d'une architecture qui tisse le lien entre la ville visible et la ville invisible.

\section{Bibliographie :}

Arendt H., La Crise de la culture. Paris, Gallimard, 1972.

Barthes R., L'Aventure sémiologique. Paris, Editions du Seuil, 1985.

Barthes Y., Callon M., Lascousmes P., Agir dans un monde incertain, Essai sur la démocratie technique, Paris, Editions du Seuil, 2001. 
Baudrillard J., La Société de consommation, Paris, Editions Denoël, 1970.

Benjamin W., L'homme, le langage et la culture, Paris, Editions Gonthier, 1974.

Bobbio N., Le futur de la démocratie, Paris, Editions du Seuil, 2007.

Cassin B., Google-moi, Paris, Editions Albin Michel, 2007.

Foucault M., Des espaces autres, Paris, Conférence au cercle des Etudes Architecturales, 14 mars 1967, in : Architecture, Mouvement, Continuité, $\mathrm{n}^{\circ} 5$ octobre 1984, pp. 46-49.

Foucault M., Surveiller et punir, Paris, Editions Gallimard, 1975.

Guillaume M., L'Empire des réseaux, Paris, Editions Descartes et $\mathrm{c}^{\mathrm{ie}}$, 1999.

Hall E. T., La Dimension cachée, Paris, Editions du Seuil, 1971.

Lapassade G., Les Microsociologies, Paris, Editions Economica, 1996.

Mac Luhan M., Pour comprendre les médias, Paris, Editions du Seuil, 1968.

Maigret E. et Mace E., Penser les médiascultures, nouvelles pratiques et nouvelles approches $d u$ monde, Paris, Editions Armand Colin. 2001.

Musso P., Télécommunications et philosophie des réseaux, la postérité paradoxale de St-Simon. Paris, PUF, 1997.

Murray P., Après l'histoire, Paris, Editons Gallimard, 2000.

Watier P., Simmel sociologue, Paris. Editions Circé, 2003.

Wolton D., Penser la communication, Paris, Editions Flammarion, 1997 